\title{
Feasibility and Safety of Stenting for Symptomatic Carotid Arterial Dissection
}

\author{
Q.Yin Y.Li X.Fan M. Ma $\quad$ G. Xu X. Liu W. Zhu \\ Department of Neurology, Jinling Hospital, Nanjing University School of Medicine, Nanjing, China
}

\section{Key Words}

Carotid artery dissection - Stent • Stroke • Transient ischaemic attack

\begin{abstract}
Objective: To determine the procedural feasibility and safety of carotid angioplasty and stent placement of carotid artery dissection (CAD) patients who had failed antithrombotic therapy in acute dissection. Methods: A series of 33 consecutive patients (mean age, 40.21 years; 29 men and 4 women) who underwent angioplasty and stent placement for symptomatic carotid artery dissection (sCAD) from May 2005 to December 2010 in the Nanjing Stroke Registry Program (NSRP) was retrospectively reviewed. All the included patients had been diagnosed definitively based on catheter angiography, and had failed to respond to medical therapy during the course of initial hospital admission. The baseline and followed up data of these patients were collected and analyzed. Results: Of all the 33 included patients, 32 patients had achieved technical success without any intraoperative complications. Two patients manifested ipsilateral Horner sign on the next day after stenting, and one of them suffered from recurrence of transient ischaemic attack (TIA) during the course of follow up, but completely remission via antithrombotic therapy. Conclusion: Endovascular stenting is a feasible and safe therapeutic strategy of CAD patients who have failed medical therapy.
\end{abstract}

Copyright $\odot 2011$ S. Karger AG, Basel

\section{Introduction}

Carotid artery dissection (CAD) refers to the intimal splitting due to a variety of pathological changes or trauma of carotid artery wall, allowing blood under arterial pressure to enter the cleft of the artery wall and split its layers, resulting in a false lumen with an intramural hematoma or aneurysmal dilatation, causing carotid stenosis, occlusion and formation of pseudoaneurysm. Either intramural hematoma or aneurysmal dilation can be sources of microemboli, and the latter can also cause mass effect on surrounding structures [1-3]. The annual incidence of spontaneous CAD is approximately 2.5 to 3 per 100,000 population, which is one of the most important causes of ischaemic stroke in the young and middle aged population, accounting for $10 \%$ to $15 \%$ of ischaemic stroke in this age group [4]. Although some studies have demonstrated that CAD patients with anticoagulant therapy had a good prognosis $[5,6]$, Beletsky found that the incidence of transient ischaemic attack (TIA), stroke or death in the warfarin anticoagulation group was $8.3 \%$ after one year followed up [7]. Moreover, Lyrer summarized several studies from different institutions, and pointed out that the incidence of death and stroke was $14.3 \%$ in the anticoagulant group of CAD [8]. The aforementioned results indicated that $\mathrm{CAD}$ with medical therapy might have a relatively high incidence of stroke. It is worth notice that anticoagulation might have a poor therapeutic effectiveness for some CAD patients with progressively aggravated neurological deficits. Additionally, surgical bypass

Wusheng Zhu

Department of Neurology, Jinling Hospital

Nanjing University School of Medicine

305 Zhongshan East Road

Nanjing 210002 (China)

Tel. +86258086 0454, Fax +86 258480 1861, E-Mail: zwsemail@gmail.com 
had been tried to perform for some CAD patients who had failed antithrombotic therapy [9], but it could not become a primary treatment of CAD due to the major procedural trauma and high-risk of complications [9, 10]. Thus, it is very important to establish a new treatment strategy for CAD.

Endovascular stent implantation had been a new therapeutic strategy for CAD over the recent years. As compared with traditional antithrombotic, stent implantation can not only help to cover the intimal cleft of the dissecting artery but also reduce thrombosis, and more importantly relieve stenosis caused by dissection. In addition, stenting may be the optimal choice for patients with progressive neurologic deficit. However, to date, only a few data and little experience can be obtained. This article summarizes the data of $33 \mathrm{CAD}$ patients with endovascular stent placement, which may help to clinical practice in the treatment of CAD.

\section{Patients and Methods}

\section{Patients}

This retrospective analysis and the protocol of this study received the approval of the local ethics committee. Written informed consent was obtained from each patient prior to the initial procedure and follow-up angiography.

From May 2005 to December 2010, a total of 33 patients with CAD that were all confirmed by DSA were selected for this study from Nanjing Stroke Registry Program (NSRP) [11]. The inclusion criteria were as follows: (1) symptomatic carotid artery dissection (sCAD) with subsequent carotid artery stenosis; and (2) patients who failed the optimal medical therapy including anticoagulation [12]. The exclusion criteria were as follows: (1) allergy to contrast agents; (2) severe bleeding tendency or severe thrombocytopenia; (3) patients who had active bleeding or surgical operations during the past 3 weeks; (4) patients who cannot tolerate the combined treatment of antiplatelet aggregation; (5) excessive tortuous access that caused operation failure; and (6) severe heart, lung or kidney failure.

\section{Procedure}

All patients were administered aspirin $100 \mathrm{mg}$ per day and Clopidogrel $75 \mathrm{mg}$ orally per day for 3 days prior to the procedure. Under a local anesthesia, an 8-French guide catheter was placed in the common carotid artery via a femoral vascular sheath. A 0.014-in microguidewire or a cerebral protection device was carefully passed through the target lesion under roadmap guidance, and the microguidewire must be ensured in the true lumen of the artery. Then stents were employed in the lesion. Multiple stents were placed over the wide-necked pseudoaneurysm until the angiography showed that the flow in the cavity of the pseudoaneurysm disappeared. After the procedure, the vascular access sheath was removed through manual femoral compression.

\section{Data collection}

Clinical and angiographic data including gender, age, risk factors for cerebrovascular disease, possible causes for CAD, admission NIHSS, radiological data like location of CAD and concurrent conditions (pseudoaneurysm), types of cerebral protection devices, types of stents, intraoperative and postoperative complications, and follow-up data were collected.

\section{Results}

The demographic, clinical and radiological data are shown in table 1 . There are 29 male and 4 female patients, ranging in age from 35 to 51 years (mean age, 40.21 years). Among the 33 patients, 27 suffered from ischaemic stroke, and 6 suffered from TIA. Five patients had a headache or neck pain prior to the onset of CAD, and 2 patients of them have a history of neck trauma. Of the 33 patients, 3 had hypertension, 2 had diabetes and no patient suffered from hyperlipidemia. A total of 2 patients have a history of smoking. The vascular inflammation indications were not found positive in all patients. All patients except one had serious stenosis $(>75 \%)$. Three cases had dissenting aneurysm; of them 1 case had bilateral CAD, and the other 2 cases had dissenting aneurysm secondary to the trauma of the common carotid artery (CCA). The lesions of CAD involved the intracranial internal carotid artery (ICA) in 1 patient, the internal carotid artery and common carotid artery at the same time in 2 patients, and the extracranial internal carotid in the other patients.

Of all the 33 patients, 32 had stents placed successfully; 1 failed stent placement because guide wire failed to cross the true lumen of vessel owing to the occlusion throughout the internal carotid artery. In the 32 patients that had stents placed successfully, a total of 45 stents were used, and cerebral protection devices were used in 13 patients. A patient who suffered from recurrent TIA prior to the operation had transient incomplete Horner sign after the operation, and the TIA was completely eliminated until double antiplatelet was administrated for more than 6 months; no new ischaemic stroke occurred in the other patients. A typical CAD with an aneurysm is shown in figure 1.

The 32 patients were followed up for the mean time of 2.3 years. Except the above-mentioned TIA patient, no patients had recurrent ipsilateral ischemic events. The DSA or CTA examinations were conducted in 15 patients on the 6 month and in 7 on the 12 months after the operation; the examinations showed that all CAD disappeared, that no restenosis occurred, and that the dissecting aneurysm disappeared in the two patients. 
Table 1. Clinical data of $33 \mathrm{CAD}$ patients

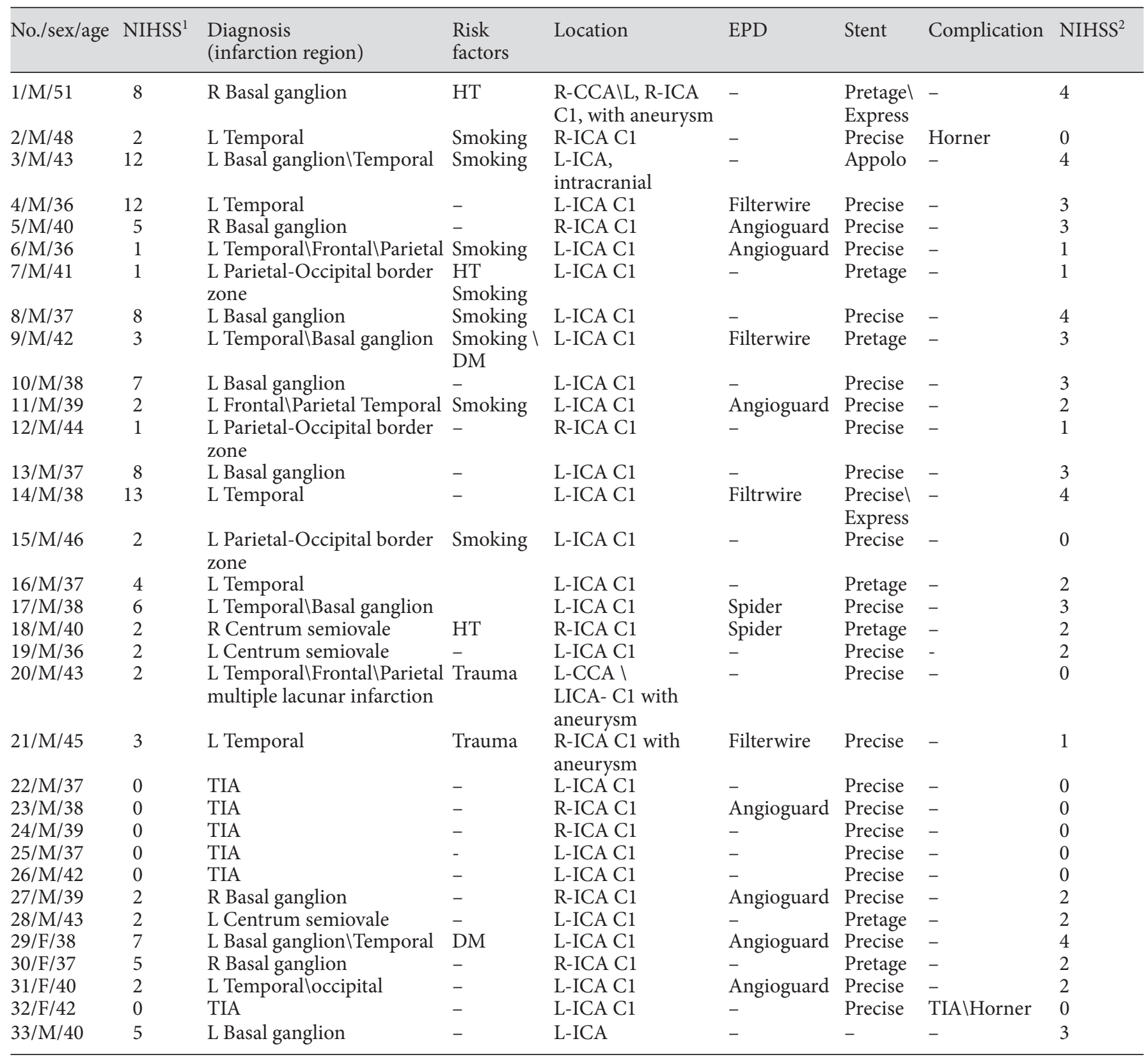

$\mathrm{CCA}=$ Common carotid artery; $\mathrm{C} 1=\mathrm{C} 1$ segment of internal carotid artery; $\mathrm{DM}=$ diabetes mellitus; $\mathrm{HT}=$ hypertension; $\mathrm{ICA}=$ internal carotid artery; L = left; NIHSS = National Institutes of Health Stroke Scale; R= right; TIA = transient ischemic attack.

NIHSS $^{1}$ the score of NIHSS on the first day of initial hospital admission. NIHSS ${ }^{2}$ the score of NIHSS on the day when discharging from hospital.

\section{Discussion}

Of all the stroke patients in USA and the European countries, 5\% are young. Nevertheless, CAD may account for about $20 \%$ of the entire population of young stroke [3]. CAD may be categorized into spontaneous, traumatic, and in addition, a few iatrogenic CAD, in according to the underlying pathogenesis. The majority of patients in this series are spontaneous except for two traumatic. The predisposed location of CAD lies in the 
Fig. 1. A typical CAD with an aneurysm demonstrated in RICA angiography of posteroanterior projection. (Patient No. 21). (a) Dissection complicated with an aneurysm (arrow) in the middle of C1 segment. (b) With two stents implantation $(8 \mathrm{~mm} \times 40 \mathrm{~mm}$, Precise), the dissection was obliterated completely and the dissecting aneurysm could be seen indistinctly (arrow). (c) After one year, the dissecting aneurysm disappeared.

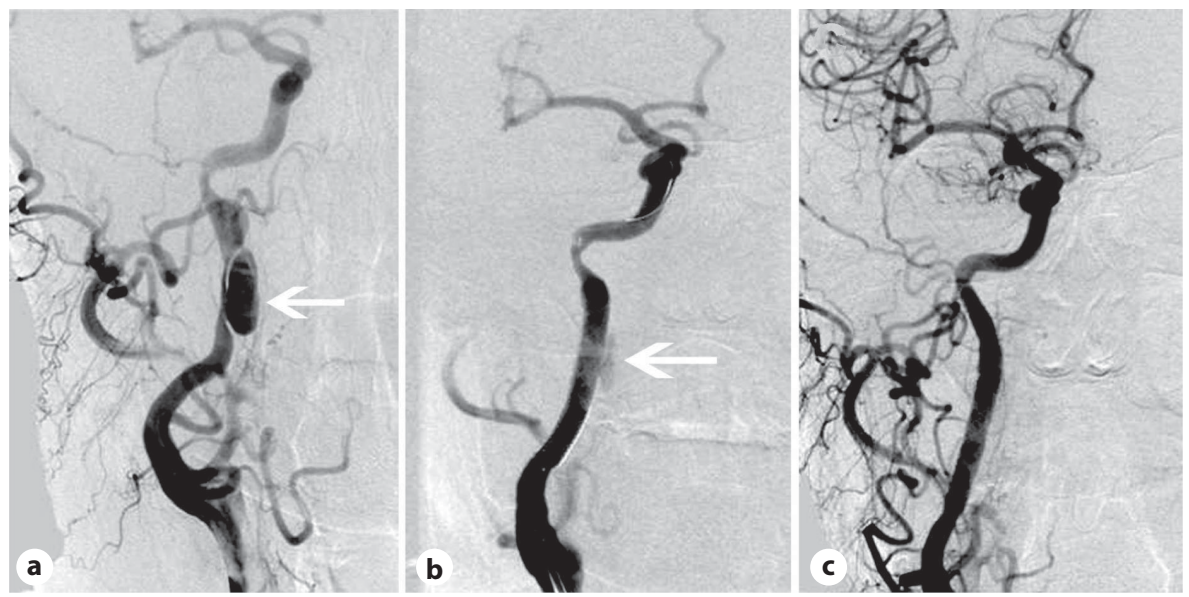

extracranial ICA. Sometimes the CCA and intracranial ICA can also be involved. Of all the 33 patients included in this series, one had intracranial ICA involved, another one was involved in CCA and ipsilateral ICA, and the remaining was involved in extracranial ICA. The clinical manifestation of spontaneous CAD mainly includes ipsilateral headache or neck pain, Horner syndrome, cerebral or ophthalmic ischaemic events. Moreover, the presentation of headache and neck pain along with Horner sign is a typical symptom of the ICA dissection. The entire spontaneous CADs in our study manifested ophthalmic or cerebral vascular ischaemic symptoms, five of which had an aura of head and neck pain during a short period of time prior to onset, but no one presented with Horner sign, which may relate to severe stenosis of carotid arteries in majority of our included subjects. Oppression on the postganglionic sympathetic fibers by local dissecting aneurysm may likely contribute to ipsilateral Horner sign [13]. Herein, one patient was complicated with a spontaneous dissecting aneurysm of extracranial ICA, and two patients manifested a traumatic dissecting aneurysm respectively. While CAD occurring, intimal of the injured artery was exposed to the following blood, which may subsequently induced thrombosis. The false lumen may extend and broaden gradually with the uninterrupted washout effects of hemodynamics, which may eventually lead to carotid artery stenosis (CAS). Thereafter, amaurosis, TIA, and ischaemic stroke may be caused due to embolism of thrombosis and CAS.

Carotid angioplasty and stenting might be considered for CAD patients when ischaemic neurological symptoms have not responded to antithrombotic therapy after acute dissection $[12,14]$. Furthermore, carotid stent imputation might also be considered for patients with extracranial CAD complicated with dissecting aneurysm [15]. All the subjects included in this study underwent stent implantation of carotid artery for the reason of their progressively aggravated neurological symptoms, despite of aggressive antithrombotic therapy for at least one week after definitive diagnosis. Of all the 33 patients, 32 had achieved technical success except one total occlusion patient failed. Of all the 32 technically successful patients, emboli protective devices (EPDs) were only used in 13 patients during procedures. There are some differences in principle of the application of EPDs between CAD and atherosclerotic carotid stenosis when implementing stent imputation, which might base on the followings. Firstly, for atherosclerotic stenosis of extracranial carotid artery, especially the lesions of carotid sinus or proximal to the sinus, the EPD is routinely deployed prior to stent implantation in order to reduce the occurrences of ischaemic stroke due to shedding of atherosclerotic debris during the procedure. Secondly, the primary involved sites of $\mathrm{CAD}$, akin to our included patients, usually locate on the section beyond the carotid sinus, which may extend from the carotid sinus to petrous segment, and the severest stenosis of the lesion generally lies in the remote of C1 segment. On condition that aforesaid, the majority of CAD patients would be lack of an appropriate place to deploy EPDs, respectively.

There was no intraoperative and postoperative occurrence of symptomatic stroke in our case series. Thirty two patients who had achieved technical success were followed up at a mean time of 2.3 years. The data of followup indicated that one CAD patient initially with a chief complain of TIA still suffered from recurrence of TIA after stenting, presenting with ipsilateral Horner sign at the same time. The patient had a significant tortuosity 
in his affected extracranial ICA before stenting, thus a vertical angle was formed on the proximal of stent after stenting. The recurrence of TIA might be associated with repeated stimulations on the intimal where carotid was angulated by the embedded stent. Meanwhile, the Horner sign might be related to that the cervical postganglionic sympathetic fibers were oppressed by the displaced ICA after stenting. Another patient was also presented with ipsilateral Horner sign. The inner lumen diameter of his affected extracranial ICA was about $6 \mathrm{~mm}$ before stenting. Excessive dilatation of local blood vessels could be observed immediately when two stents $(8 \mathrm{~mm} \times 40 \mathrm{~mm}$, Precise $\left.^{\mathrm{Tw}}\right)$ were embedded into $\mathrm{C} 1$ segment of the affected ICA. Horner sign appeared on the next day after stenting, which might be associated with the continuing oppression on the cervical postganglionic sympathetic fibers by the excessive dilated ICA. Based on the status of the target vascular diameters altered preoperatively and postoperatively, we considered that the underlying pathogenesis of this CAD patient might be fibromuscular disease (FMD). We may acquire the following learning points from the above-mentioned two patients. First, a stiffer stent should avoid being implanted into a tortuous ICA. Second, a suitable stent should be selected as possible as to match the inner lumen diameter of target artery.

Although the presenting descriptive study have some limitations such as single-center, small samples, retrospective and non-randomized, the result indicated that the majority of CAD patients who had not responded to antithrombotic therapy might benefit from carotid angioplasty and stenting, and the safety of the procedure is fairly satisfactory.

\section{Acknowledgements}

This study was supported by Natural Science Foundation of China (NSFC \#30870847 to XL and NSFC \#30870848 to GX).

\section{Disclosure Statement}

The authors had no conflicts of interest to declare.

\section{References}

$D_{1}$ Edgell RC, Abou-Chebl A, Yadav JS Endovascular management of spontaneous carotid artery dissection. J Vasc Surg 2005;42: 854-860.

2 Kadkhodayan Y, Jeck DT, Moran CJ, Derdeyn $\mathrm{CP}$, Cross DT 3rd: Angioplasty and stenting in carotid dissection with or without associated pseudoaneurysm. AJNR Am J Neuroradiol 2005;26:2328-2335.

-3 Kittner SJ: Stroke in the young: coming of age. Neurology 2002;59:26-33.

4 Wouter I, Schievink WI: Spontaneous dissection of the carotid and vertebral arteries. N Eng J Med 2001;344:898-906.

5 Shah Q, Messe SR: Cervicocranial arterial dissection. Curr Treat Options Neurol 2007; 9:55-62.

6 Cohen JE, Gomori JM, Umansky F: Endovascular management of symptomatic vertebral artery dissection achieved using stent angioplasty and emboli protection device. Neurol Res 2003;25:418-422.
Beletsky V, Nadareishvili Z, Lynch J, Shuaib A, Woolfenden A, Norris JW, et al: Cervical arterial dissection: time for a therapeutic trial? Stroke 2003;34:2856-2860.

8 Philippe L, Stefan E: Antithrombotic drugs for darotid artery dissection. Stroke 2004;35: 613-614.

9 Müller BT, Luther B, Hort W, NeumannHaefelin T, Aulich A, Sandmann W: Surgical treatment of 50 carotid dissections: indications and results. J Vasc Surg 2000;31:980988.

10 Morgan MK, Sekhon LH: Extracranialintracranial saphenous vein bypass for carotid or vertebral artery dissections: a report of six cases. Journal of Neurosurgery 1994;80:237246.

$11 \mathrm{Xu}$ G, Liu X, Wu W, Zhang R, Yin Q: Recurrence after ische patients: impact of uncontrolled modifiable risk factors. Cerebrovasc Dis 2007;23:117-120.
2 Brott TG, Halperin JL, Abbara S, Bacharach JM, Barr JD, Bush RL, et al: Guideline on the Management of Patients With Extracranial Carotid and Vertebral Artery Disease. J Am Coll Cardiol 2011;57:1002-1044.

13 Huang Y, Zhang S: Horner syndrome. Chinese Journal of Clinical Anatomy 2008;6:696-699.

14 Gonzales-Portillo F, Bruno A, Biller J: Outcome of extracranial cervicocephalic arterial dissections: a follow-up study. Neurol Res 2002;24:395-398.

15 Albuquerque FC, Han PP, Spetzler RF, Zabramski JM, Mcdougall CG: Carotid dissection: technical factors affecting endovascular therapy. Can J Neurol Sci 2002;29: 54-60. 